${ }^{2}$ Clinical Professor, Department of Psychiatry, Cooper

Medical School of Rowan University, Camden, NJ

${ }^{3}$ Clinical Faculty, St. Elizabeths Hospital, DBH,

Washington, DC

ABSTRACT: Delusional misidentification syndromes are rare psychotic disorders, in which the patient believes that the identity of a person, object, or place has been changed or replaced by another. These disorders include Capgras syndrome, Fregoli syndrome, and intermetamorphosis. Reverse types of misidentification syndromes were introduced to distinguish alteration of the self rather than of others.

Reverse Capgras syndrome refers to the psychological change of the self as opposed to others, while reverse Fregoli syndrome is the delusional belief in which the patient has undergone fundamental changes in physical makeup without any psychological changes, and reverse intermetamorphosis is a variant in which patients believe that they have undergone physical and psychological transformation.

Here we present an interesting case of a transgender woman presenting with delusional misidentification of the self in the context of Schizophrenia. A review of literature, with emphasis on etiological factors, forensic implications and association with violence is presented.

FUNDING ACKNOWLEDGEMENTS: No funding.

199

\section{Cardiac Safety and QTc Intervals With DM/Q Treatment for Pseudobulbar Affect: A Review of Clinical Trial Data}

Jay W. Mason, MD'; Emmanuelle Hugentobler, $\mathrm{MD}^{2}$; Andrea E. Formella, PharmD ${ }^{2}$; and Laura E. Pope, PhD

${ }^{1}$ Mason Cardiac Safety Consulting, Reno, NV

${ }^{2}$ Avanir Pharmaceuticals, Inc., Aliso Viejo, CA

ABSTRACT: OBJECTIVE: Dextromethorphan hydrobromide and quinidine sulfate (DM/Q) $20 \mathrm{mg} / 10 \mathrm{mg}$ is FDA approved to treat pseudobulbar affect (PBA), a neurological condition characterized by sudden, frequent, involuntary crying or laughing. Although the total dose of quinidine $(20 \mathrm{mg})$ from twice daily DM/Q for PBA is well below the antiarrhythmic dose (600-1600 mg/day), clinicians may be reticent to use DM/Q due to concerns for cardiac safety.
METHOD: DM/Q cardiac safety was evaluated in two thorough QTc (TQT) studies and by ECG monitoring in DM/Q phase 3 clinical trials. In the TQT studies, twice daily DM/Q 30mg/10mg (Study 08-AVR-126; N=50 enrolled) and supratherapeutic doses $30 \mathrm{mg} / 30 \mathrm{mg}$ and $60 \mathrm{mg} / 60 \mathrm{mg}$ (Study 05-AVR-119; N = 36 enrolled) were studied in healthy volunteers. In phase 3 controlled clinical trials, the effects of DM/Q on Fridericia's corrected QT intervals $(\mathrm{QTcF})$ were assessed, as was the incidence of ECG outliers. Adverse events (AEs) were monitored in all clinical trials.

RESULTS: Overall, 47/50 participants completed TQT study 08-AVR-126 and 36/36 completed TQT study 05-AVR-119. Time-matched, placebo-corrected mean maximal changes in QTcF for DM/Q $30 \mathrm{mg} / 10 \mathrm{mg}$ and $30 \mathrm{mg} / 30 \mathrm{mg}$ occurred $3 \mathrm{~h}$ post-dose ( 10.3 and $10.1 \mathrm{~ms}$, respectively) vs moxifloxacin $(12.2$ and $14.4 \mathrm{~ms}$ at 1.5 and $1.0 \mathrm{~h})$. For the supratherapeutic DM/Q 60/60 mg dose, mean maximal QTcF change was $18.8 \mathrm{~ms}$. No participant had a QTcF $>480$ ms or QTc increase $>60 \mathrm{~ms}$. In PBA phase 3 controlled trials, mean changes in QTcF were similar for DM/Q containing Q $10 \mathrm{mg}$ (0.4 to $3.5 \mathrm{~ms} ; \mathrm{n}=217)$ andplacebo (0.4 to $3.1 \mathrm{~ms} ; \mathrm{n}=183$ ), but greater for $\mathrm{DM} / \mathrm{Q}$ with $\mathrm{Q} 30$ $\mathrm{mg}$ (2.9 to $7.6 \mathrm{~ms} ; \mathrm{n}=146)$. In an outlier analysis, a similar percentage of DM/Q-treated participants $(3.9 \%)$ had a QTcF shift from $<450 \mathrm{~ms}$ at baseline to $\geq 450 \mathrm{~ms}$ during treatment vs placebo $(2.9 \%)$. No participant with PBA had a QTc change from baseline $>60 \mathrm{~ms}$ or an absolute QTc interval $>480 \mathrm{~ms}$. No dose- or time-related trends in cardiac arrhythmias or other cardiac-related AEs were observed. Of $2552 \mathrm{DM} / \mathrm{Q}$-treated patients and healthy participants across all controlled and open-label trials for any indication, 11 deaths due to any cardiovascular $\mathrm{AE}$ have been reported; none were attributable to DM/Q treatment and none occurred in placebo-controlled PBA clinical trials in any treatment group. Overall 16 patients had a QTcF that exceeded $500 \mathrm{~ms}$ at any ECG measurement.

CONCLUSIONS: The TQT studies demonstrated that DM/Q has the potential to prolong the QT interval in a dosedependent manner, but that the risk for QTc prolongation and arrhythmias with Q $10 \mathrm{mg}$ formulations is low. In clinical trials with PBA patients, the cardiac safety profile of $\mathrm{DM} / \mathrm{Q} 20 \mathrm{mg} / 10 \mathrm{mg}$ or $30 / 10 \mathrm{mg}$ was indistinguishable from placebo.

FUNDING ACKNOWLEDGEMENTS: Avanir Pharmaceuticals, Inc. 